\title{
A cadaveric analysis of contact stress restoration after osteochondral transplantation of a cylindrical cartilage defect
}

\author{
Niels B. Kock • José M. H. Smolders • \\ Job L. C. van Susante $\cdot$ Pieter Buma • \\ Albert van Kampen · Nico Verdonschot
}

Received: 1 October 2007/Accepted: 21 January 2008/Published online: 22 February 2008

(C) The Author(s) 2008

\begin{abstract}
Osteochondral transplantation is a successful treatment for full-thickness cartilage defects, which without treatment would lead to early osteoarthritis. Restoration of surface congruency and stability of the reconstruction may be jeopardized by early mobilization. To investigate the biomechanical effectiveness of osteochondral transplantation, we performed a standardized osteochondral transplantation in eight intact human cadaver knees, using three cylindrical plugs on a fullthickness cartilage defect, bottomed on one condyle, unbottomed on the contralateral condyle. Surface pressure measurements with Tekscan pressure transducers were performed after five conditions. In the presence of a defect the border contact pressure of the articular cartilage defect significantly increased to $192 \%$ as compared to the initially intact joint surface. This was partially restored with osteochondral transplantation (mosaicplasty), as the rim stress subsequently decreased to $135 \%$ of the preoperative value. Following weight bearing motion two out of eight unbottomed mosaicplasties showed subsidence of
\end{abstract}

N. B. Kock · J. M. H. Smolders · P. Buma - N. Verdonschot Orthopaedic Research Laboratory, Department of Orthopaedics, Radboud University Nijmegen Medical Centre,

P.O. Box 9101, Nijmegen, The Netherlands

e-mail: smolders_jose@hotmail.com

J. L. C. van Susante $(\square)$

Department of Orthopaedics, Rijnstate Hospital,

Postbus 9555, 6800 TA Arnhem, The Netherlands

e-mail: jvansusante@alysis.nl

A. van Kampen

Department of Orthopaedics, Radboud University Nijmegen

Medical Centre, Nijmegen, The Netherlands the plugs according to Tekscan measurements. This study demonstrates that a three-plug mosaicplasty is effective in restoring the increased border contact pressure of a cartilage defect, which may postpone the development of early osteoarthritis. Unbottomed mosaicplasties may be more susceptible for subsidence below flush level after (unintended) weight bearing motion.

Keywords Biomechanics · Cartilage ·

Articular/pathology · Humans · Knee Joint/Surgery ·

Pressure - Surface Properties - Transplantation .

Autologous $\cdot$ Weight-bearing

\section{Introduction}

Full-thickness cartilage defects may lead to early osteoarthritis [6,13,14]. Osteochondral transplantation is a successful treatment for these defects, but the outcome is dependent on, among other parameters, stability and restoration of surface congruency $[9,15,21]$. In our previous study about the effect of perfect depth alignment of a transferred plug [8], we evaluated the difference in stability between bottomed plugs (donor plug length and recipient defect depth accurately matched) and unbottomed plugs (donor plug is left shorter than the recipient defect depth). That study demonstrated that bottoming plugs resulted in a significantly more stable situation than unbottoming plugs. Unbottomed plugs leave a cavity at the bottom of the defect and therefore rely exclusively on frictional forces. When performing only a single-plug transfer the whole plug is, in most cases, nicely surrounded by (subchondral) recipient bone, and stability is fairly good, bottomed or not. However, when performing osteochondral transplantation where more than one plug is needed, stability is probably lower because of gaps between 
the round plugs and their surrounding bone. Kordas et al. [10] reported in agreement with this that the push-in force below flush level for (unbottomed) multiple grafts was significantly lower compared to a single graft. Theoretically, width differences of the cartilage layer between trochlea (donor area) and condyl (defect area) might have an influence on restoration of surface congruency.

Usually a patient is restricted in weight bearing after osteochondral transplantation. Rehabilitation periods of two weeks of non-weight bearing and an additional two to three weeks of partial weight bearing are reported in the literature [6]. This period facilitates good ingrowth of (subchondral) bone $[11,15]$ and thus ensures the preservation of surface congruency as it was intended directly postoperative. Histological research has proven that after transplantation bone resorption takes place on the recipient site and on the graft surface [5,18]. This might be one reason for a gradual loss of the initial press-fit stability postoperatively and a cause of subsiding below flush level of the graft during follow-up. Accordingly, Whiteside et al. [20] reported a reduction in short-term load bearing capacity of a single-plug transplant one week after transplantation. Press-fit mechanisms provide stability up to 4 weeks [6]; subsequently, this is taken over by the integration of the graft and the recipient bone [11].

The objective of the present study was to investigate the biomechanical effectiveness of osteochondral transplantation. More specifically we assessed whether the treatment would decrease peak stresses at the boundaries of the original articular cartilage defect. Subsequently, we analysed if articular stresses were dependent on plug placement (bottomed versus unbottomed) and how this was affected by loads that represented (for instance unintended) early weight bearing.

\section{Materials and methods}

\section{Materials}

Eight intact human cadaver knees obtained from the anatomical department, from individuals 70-80 years old of unknown gender, were used. Exclusion criteria were severe arthrosis of donor or graft area and malignant processes within the knee. Specimens were freshly frozen and thawed at room temperature overnight before preparation and testing. The K-scan 4000 (Tekscan Inc., Boston, MA) (Fig. 1) was used for measuring surface congruency according to a previously discussed protocol [4]. Before testing, each new Tekscan sensors was pre-loaded and calibrated, as suggested by the Tekscan manual and by Brimacombe et al. [2]. For this loading, a circular plastic plunger, that almost covered the entire width of the sensor,

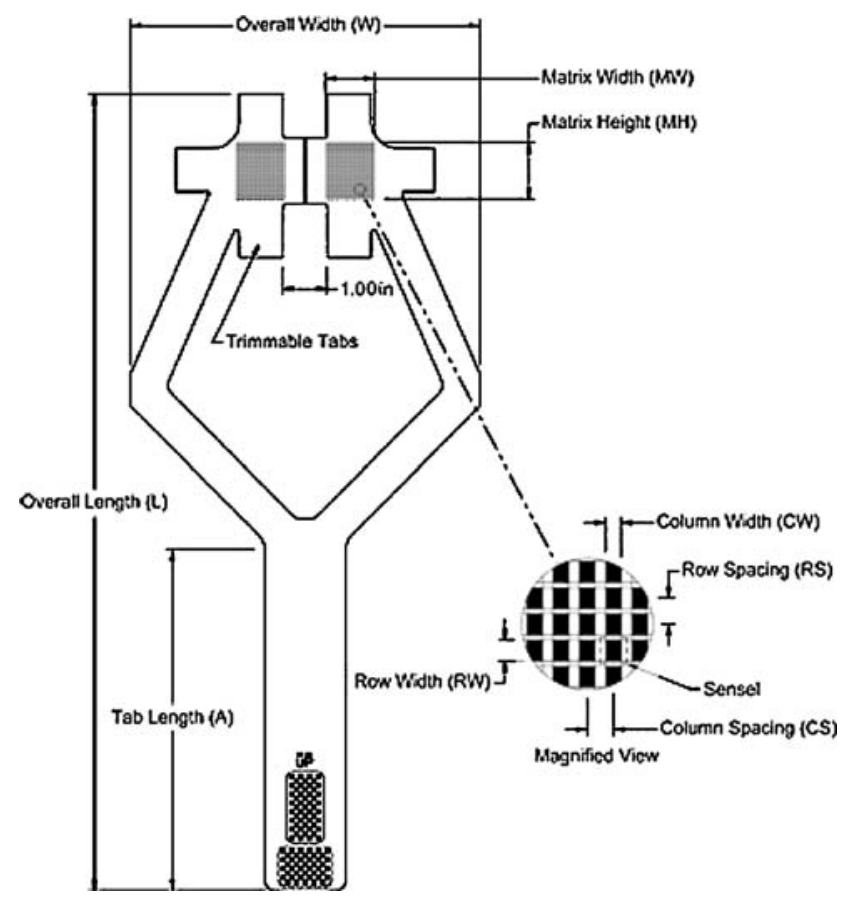

Fig. 1 Tekscan K-4000 measuring device with two thin $(0.089 \mathrm{~mm})$ and flexible pressure sensors, which were able to conform to the anatomy of the medial and lateral knee compartments. The sensors consist of printed circuits divided into grids of load-sensing regions. Each sensor measured $28 \times 33 \mathrm{~mm}$, with a total of 572 pixels with a surface per pixels of $1.27 \mathrm{~mm}^{2}$

was placed underneath a small loading platform where weights could be placed on top. Each sensor was calibrated separately and calibration files were stored. A new sensor was used for each knee. The sensor measurements were presented as a 22 by 26 pixel matrix with square pixels. For the osteochondral transplantations, a disposable $8 \mathrm{~mm}$ Osteochondral Autograft Transfer System (OATS) was used (Arthrex, Somas, Sint Anthonis, The Netherlands).

\section{Preparations}

Skin, muscles, excess soft tissues, patella and the anterior part of the knee capsule were removed from each knee, whereas collateral ligaments and intra-articular structures were left intact. In this way the tibio-femoral joint area was uncompromised and yet was accessible from the anterior side. Dorsally two small 'windows' were created in the capsule, giving access to the posterior part of both condyles. The femur as well as the tibia and fibula were sectioned at $\sim 18 \mathrm{~cm}$ from the joint space. The knee was then placed in a knee-testing device, which was used in earlier experiments [1,19] (Fig. 2). This device provides five degrees of freedom of motion for the tibial side, whereas the femoral side has one degree of freedom and can additionally be manually displaced for flexing or 

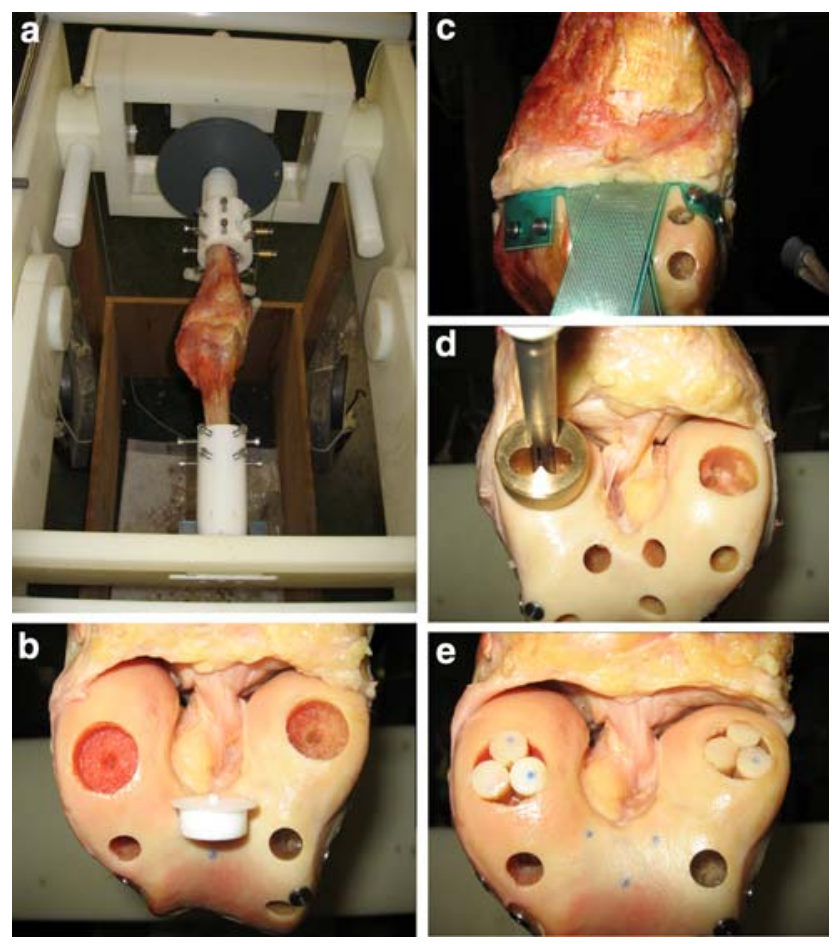

Fig. 2 a Knee-testing device. b Circular plastic cap for positioning of Tekscan sensor. c Tekscan sensor in situ. d Special mould for OATS. e Three-plug mosaicplasty placed flush in full-thickness osteochondral defect

extending movements of the knee (Fig. 2). Weights could be attached to the femur side such that the force may be directed through the longitudinal axis of the femur. To simulate partial weight bearing, we applied $350 \mathrm{~N}$ as a tibio-femoral compressive force. Previous research at our institution has shown that a force of $171 \mathrm{~N}$ (unbottomed) and $384 \mathrm{~N}$ (bottomed) was necessary to push a single-plugmosaicplasty below flush-level [8].

\section{Operation and testing}

First, donor sites were marked with a permanent marker on the trochleae or the posterior region of the condyles. Three plugs were harvested with a length of $\sim 15 \mathrm{~mm}$ (to be unbottomed) and three plugs of $\sim 18 \mathrm{~mm}$ length (to be bottomed). After harvesting all six donor plugs, Tekscan sensors were placed inside the tibio-femoral joint space. After positioning the knee in approximately $45^{\circ}$ flexion, the sensors were guided alongside the cruciate ligaments (one on each side), and placed between each femoral condyle and tibial plateau. Subsequently, a small circular plastic cap (diameter $10 \mathrm{~mm}$, height $1 \mathrm{~mm}$ ) was pinned on both condyles, at the location where the mosaicplasty was to be performed (Fig. 2). The compression of both markers on the sensors was clearly visible on the computer screen and assisted in positioning the sensors centrally over the area where the mosaicplasty would be executed. To facilitate repositioning of the sensors between different interventions, the sensors were fixated to the joint by four small metal 'anchors' on all four corners of the sensor that could be used to re-attach the sensors to the joint (Fig. 2). In order to confirm validity of this method a test was performed where the sensor was repeatedly fixated and completely removed from the knee and the plastic marker was visualized. Reproducibility of the re-attachement of the sensor was confirmed by five independent measurements of the position of a circular cap, each after removing a re-attachment of the Tekscan sensor. Four out of five measurements showed exactly the same position of the cap within the Tekscan matrix, which proves a high accuracy of re-attachment.

To investigate the consequences of a cartilage defect and subsequent mosaicplasty on the stress distribution under various conditions, Tekscan recordings were performed under five different conditions: (A) preoperatively, intact condition, (B) cartilage defect without any reconstruction, (C) following mosaicplasty, (D) after mosaicplasty and subsequent non-weight bearing motion of the knee and (E) after mosaicplasty with weight bearing motion (E). All surface measurements were performed with the knee in $0^{\circ}$ extension, thereby ensuring loading of the (restored) defective sites, and an axial load of $350 \mathrm{~N}$.

The 'preoperative' measurement (A) was with intact condyles. After removing the sensors, both on the medial and lateral condyle a standardized subchondral defect, $8 \mathrm{~mm}$ deep was created by a circular drill of $16 \mathrm{~mm}$ diameter, the center being at exactly the same location as the center of the plastic marker that was measured previously.

The 'defect' measurement (B) of the condyle surfaces was performed after creating the osteochondral defect. With the use of a special mould (Fig. 2) and the OATS, these defects were prepared to receive three osteochondral plugs. When the mosaicplasty was to be performed as bottomed, three defects were created, approximately $15 \mathrm{~mm}$ deep and the bottoms of the defects were tamped. Following measuring of the depths of these defects, three of the 18-mm donor plugs were matched for these depths by carefully removing some subchondral bone with surgical bone-nibbling pliers according to clinical practice. Plugs were orientated such that the most congruent surface would be achieved. These plugs were tampered in place, until flush level of the cartilage surface with the recipient site was achieved (Fig. 2). At the opposite condyle, which was to be unbottomed, three defects of approximately $20 \mathrm{~mm}$ depth were created, and the donor plugs of $15 \mathrm{~mm}$ were used. The difference of $5 \mathrm{~mm}$ in length between the defects was essential for the unbottomed plugs to be sure to have a cavity at the bottom of the defect and therefore rely exclusively on frictional forces. 
Fig. 3 a Typical example of Tekscan report on load distribution for a cartilage defect without any reconstruction. b The mosaicplasty area (region 1) is a circle consisted of square pixels with a diameter of 12 pixels. The border area (region 2) is a ring with a diameter of 3 pixels around the defect circle. A transition zone of 1 pixel around the mosaicplasty area is found between regions 1 and 2
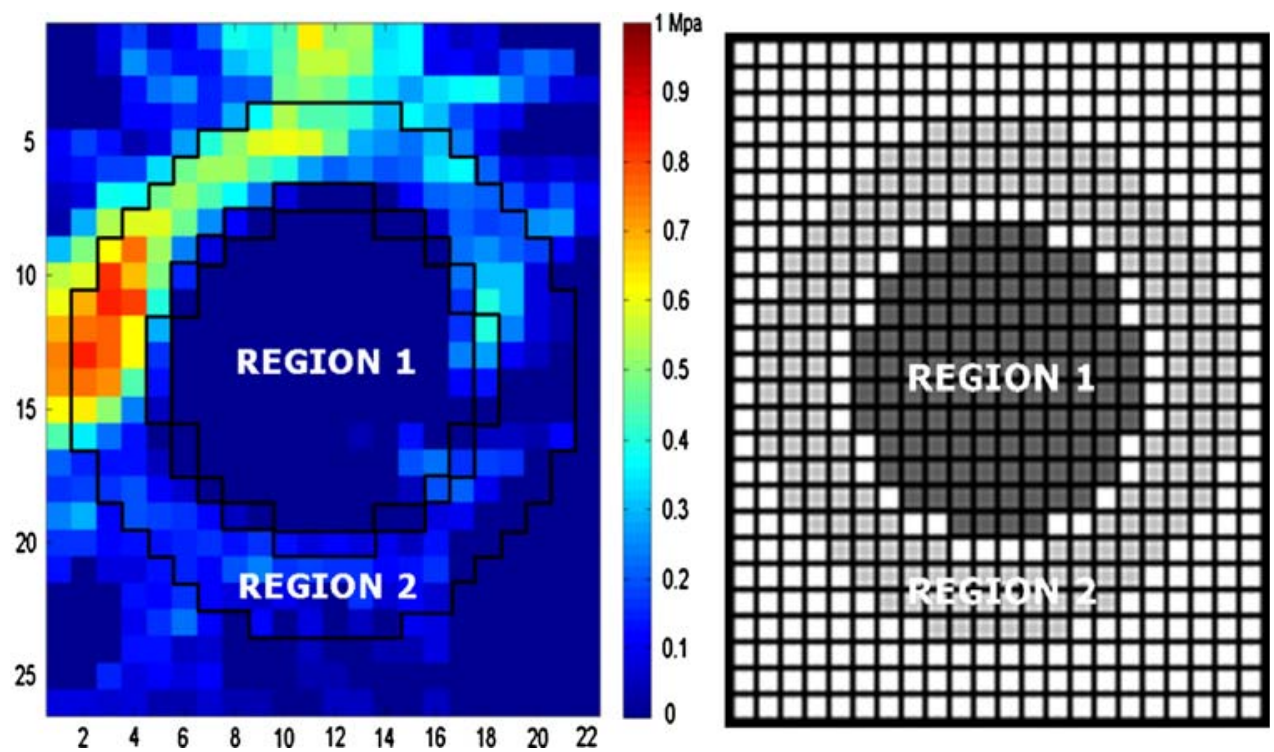

The 'mosaicplasty' surface measurement (C) was executed after performing both mosaicplasties. The 'nonweight bearing motion' measurement (D) was performed following flexing and extending the knee 20 times, while no weight was attached to the test device. The axial load of $350 \mathrm{~N}$ was re-applied before the measurement was performed. The final 'weight bearing motion' measurement (E), was performed once the knee had been flexed and extended 20 times with $350 \mathrm{~N}$ of axial force applied to the knee while performing the flexion-extension movements.

\section{Evaluation of variables}

Each of the eight knees provided two paired standardized mosaicplasties of three plugs, one bottomed and one unbottomed. Thus, there were 16 mosaicplasties: eight bottomed and eight unbottomed. Three general groups were created for statistical evaluation: the whole group of all mosaicplasties (bottomed and un-bottomed combined), a group with only bottomed and one with only unbottomed mosaicplasties. The five measurements performed were: (A) preoperative, (B) defect, (C) mosaicplasty, (D) nonweight bearing motion, (E) and weight bearing motion.

Relative to the reconstructed surface, we defined two specific regions of interest: the mosaicplasty area itself and the border of the reconstructed surface. The first region obviously quantified the amount of stress transferred at the mosaic site, whereas the second region was selected to quantify the amount of stress transferred to the border of the defect. The mosaicplasty area (region 1) was a circle with a diameter of $16 \mathrm{~mm}$, for which the best fitting circle representing the standardized defect was obtained on the Tekscan sensor matrix (Fig. 3). This circle was obtained by calculating the lowest surface pressure of a 16-mm diameter circle in the non-reconstructed (defect) case. The border region (region 2) was selected around the first region and had a width of 3 pixels $(3.81 \mathrm{~mm})$. To ensure that the two regions were clearly separated a transition zone with a width of 1 pixel was selected and has not included in the measurements (Fig. 3). The total of the pixel values in regions 1 and 2 , respectively, were used for further comparative evaluations. To allow for a direct comparison of the pressure values, the pressures on the border and mosaic areas were normalized to the total force on the sensor.

\section{Statistics}

Contact pressures were calculated at the boundaries of the cartilage defect (region 2) as well as for the defect/mosaicplasty (region 1) itself. This was done for five different conditions as described before (A-E). These values were expressed for three groups, namely bottomed, unbottomed and combined.

Differences were examined within and among the groups. For statistical analysis, we used SPSS 12.0 for Windows. The linear mixed model was used to evaluate the influence of bottoming or unbottoming the plugs, with Bonferroni correction. $P<0.05$ were considered to be statistically significant.

\section{Results}

The averaged contact-pressures at the border contact surface are shown in Fig. 4. Overall, there were no significant differences between bottomed and unbottomed. All the 


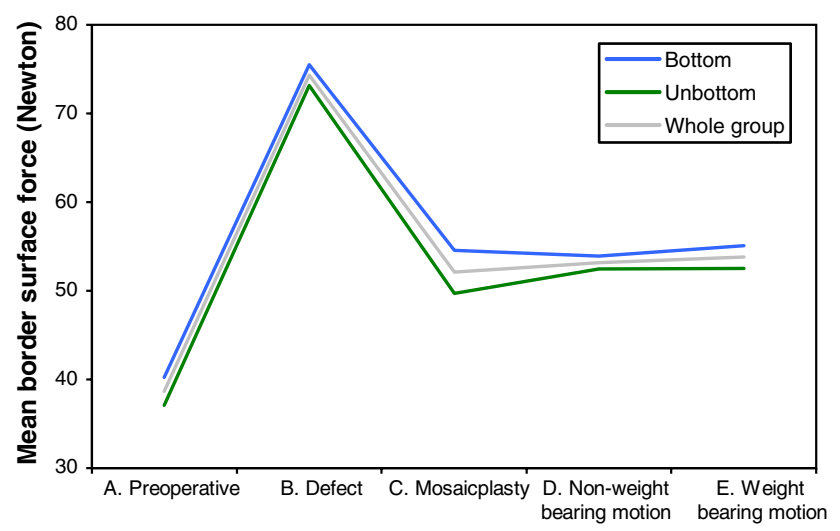

Fig. 4 Graph showing the border contact pressure for three groups; bottomed (blue line), unbottomed (green line) and the whole group (grey line). In presence of a defect (b) the border contact surface pressure was significantly higher compared to the intact (preoperative) situation (a), performing a mosaicplasty made the border pressure decline to approach preoperative values (c), and after motion with and without weight the border contact pressure remained stable (c-e). Standardized deviation values are presented in Table 1

data showed the same pattern for the three groups: in presence of a defect (B) the border contact surface pressure was significantly higher compared to the intact (preoperative) situation (A), performing a mosaicplasty made the border pressure decline to approach preoperative values (C), and after motion with and without weight the border contact pressure remained stable (C-E).

The pressure pattern of the mosaic contact surface was inverse to the border contact surface pressure (Fig. 5): the mosaicplasty area had almost no contact pressure after creating a defect (B), in the presence of the mosaicplasty the contact surface pressure was regained to some extent

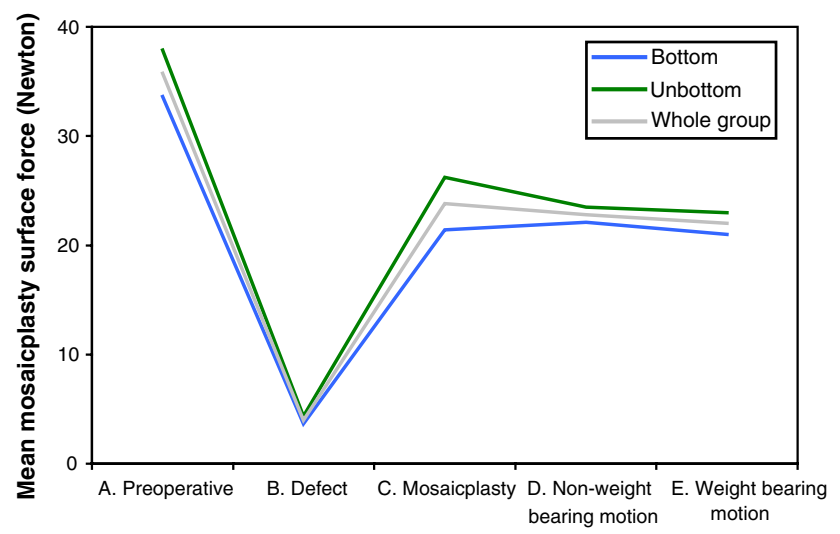

Fig. 5 Graph showing the mosaicplasty contact pressure for three groups; bottomed (blue line), unbottomed (green line) and the whole group (grey line). The mosaicplasty area had almost complete lack of contact pressure after creating a defect (b), in presence of the mosaicplasty the contact surface pressure was regained (c vs. a) and the contact pressure remained stable after non-weight bearing and weight bearing motion (c-e). Standardized deviation values are presented in Table 1
(C vs. A) and the contact pressure remained relatively constant after non-weight bearing and weight bearing motions (C-E).

Two out of eight unbottomed versus zero bottomed mosaicplasties showed a decreased mosaicplasty pressure and increased rim stress after weight bearing motion on individual Tekscan measurements, indicating that the mosaicplasty subsided below flush level. Five out of eight bottomed plugs were placed on the medial condyle and no significant differences in pressure or rim stress was found.

\section{Statistics}

Mean pressure values measured are given in Table 1 .

Effect of defect $(A-B)$ : Pressure redistribution occurred in the presence of a defect from the mosaic contact surface to the cartilage surrounding it. The border contact pressure increased to $192 \%(P=0.000)$ compared to the preoperative border contact surface pressure, while the pressure on the defect contact surface decreased to $11 \%(P=0.000)$.

Effect of mosaicplasty $(B-C)$ : The mean stress elevation around the defect was partially restored in the presence of a mosaicplasty, as the border contact pressure decreased by $30 \%$ compared to the defect measurement. This resulted in a border contact pressure of $135 \%$ compared to intact cartilage. The mosaic contact pressure was restored from 11 to $67 \%(P=0.000)$.

Effect of flexion-extension motion $(C-D-E)$ : The border contact pressure did not return to preoperative values after performing a mosaicplasty, and did not change after flexion and extension motions. The border contact pressure after non-weight bearing motion' and 'weight bearing motion' remained equal to the mosaicplasty measurement, 138 and $139 \%$ ( $P=0.001$ and 0.000 , respectively) compared to intact, preoperative cartilage. For these measurements the mosaic contact pressures remained lower than those of intact cartilage, with pressures 64 and $62 \%$, respectively (respectively $P=0.001$ and 0.001 ).

\section{Discussion}

In this biomechanical human cadaver study we clearly demonstrated that an osteochondral cartilage defect severely affects the contact pressure on the remaining intact joint surface. Obviously, there are some additional limitations to our study. The study had only a limited number of knees. During testing osteoarthritic changes in bone were found in some of these elderly knees. Clearly this is different to the bone quality of the typical patient who is relatively young and active. Another limitation was that during the preparations the knee was positioned in 
Table 1 Results of the surface contact pressure measurements of five conditions a Values are given as mean (SD), only significant $P$-values are shown

\begin{tabular}{llllr}
\hline Measurement & $\begin{array}{l}\text { Whole } \\
\text { group } \\
(n=16)\end{array}$ & $P$-value & $\begin{array}{l}\text { Bottomed } \\
(n=8)\end{array}$ & $\begin{array}{l}\text { Unbottomed } \\
(n=8)\end{array}$ \\
\hline Border & & & & \\
A. Preoperative & $38.7(7.1)^{\mathrm{a}}$ & & $40.2(8.3)$ & $37.1(6.0)$ \\
B. Defect & $74.3(5.5)$ & (B vs. A-C-D-E) 0.000 & $75.5(3.9)$ & $73.2(6.8)$ \\
C. Mosaicplasty & $52.1(9.3)$ & (A vs. C) 0.001 & $54.6(7.5)$ & $49.7(10.7)$ \\
D. Motion without weight & $53.2(9.8)$ & (A vs. D) 0.001 & $53.9(8.4)$ & $52.4(11.6)$ \\
E. Motion with weight & $53.8(9.6)$ & (A vs. E) 0.000 & $55.1(9.0)$ & $52.5(10.6)$ \\
Mosaic & & & & \\
A. Preoperative & $35.7(7.8)$ & & $33.6(9.3)$ & $37.9(5.6)$ \\
B. Defect & $4.0(4.0)$ & (B vs. A-C-D-E) 0.000 & $3.7(3.8)$ & $4.3(4.4)$ \\
C. Mosaicplasty & $23.8(8.2)$ & (A vs. C) 0.002 & $21.4(7.4)$ & $26.2(8.9)$ \\
D. Motion without weight & $22.8(8.7)$ & (A vs. D) 0.001 & $22.1(8.1)$ & $23.5(9.7)$ \\
E. Motion with weight & $22.0(9.1)$ & (A vs. E) 0.001 & $21.0(8.4)$ & $23.0(10.1)$ \\
\hline
\end{tabular}

such a way that both condyles would be loaded with approximately the same force, which may be different from in vivo loads. This might have had an influence on the absence of medial/lateral differences. Contrary to other experiments we kept all collateral ligaments and intraarticular structures intact, which resembles the anatomical situation more closely and thus gives a greater translational value of pressure transfer. Clearly, no biological effects were taken into account such as the resorption of the plugs (thereby reducing the stability) or bony ingrowth (thereby enhancing stability). We also measured the contact patterns under static conditions with the knee in extension, whereas in reality shear forces at different flexion angles are also applied to the reconstruction. These limitations should be taken into account when interpreting the results.

The results showed that the average border contact pressure almost doubled (increase of 92\%) compared to the pressure on an intact congruent joint surface. In the literature, increases in peak pressure between 10 and $30 \%$ were found in the presence of a defect with a diameter ranging

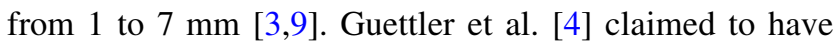
found no difference in rim contact pressure for defects below $10 \mathrm{~mm}$ diameter, but they found a $64 \%$ increase with respect to the healthy situation for all defects above $10 \mathrm{~mm}$. These findings are supported with a recent finite element model, in which it was found that large defects (greater than $0.78 \mathrm{~cm}^{2}$ ) resulted in significantly increased border contact surface pressures, which may have clinical implications [16].

The relation between increased contact stresses on the joint surface and progressive degenerative changes of the cartilage is well recognized. Lefkoe et al. found a significant decrease in proteoglycan content in the cartilage sampled from the rim of 20-week-old defects [12]. Jackson et al. [7] introduced a 'zone of influence', as he found cartilage adjacent to the defect being affected, which may lead to early secondary gonarthrosis. Messner and Maletius [14] reported in a follow-up study radiographic joint space reduction in almost $50 \%$ of the patients with severe cartilage damage 14 years earlier. Linden et al. [13] found in a follow-up study of 33 years that $80 \%$ of the adults with osteochondritis dissecans developed secondary gonarthrosis. The gonarthrosis seemed to have its onset 10 years earlier (mean age 49 instead of 59 years) in life than primary gonarthrosis. Clearly, the increased potential of degenerative changes in the knee following an articular cartilage defect is of great clinical importance. Especially because this type of cartilage defect frequently occurs in the younger aged population.

In our study we were able to reduce the contact pressure at the boundaries of a large articular cartilage defect by $30 \%$ with a osteochondral transplantation. In a study by Raimondi et al. [17] a $16 \%$ reduction in peak pressure was found in the presence of a fibrin glue graft. One could question as to what extent this reduction lasts since fibrin glue degrades quite rapidly. Koh et al. [9] reported peak contact pressure reduced to normal when plugs were flush. However, they used only one plug, which does not correspond to clinical practice. It may be more difficult to obtain a smooth cartilage surface when using more plugs, which explains why we did not find a complete normalization of the contact stresses.

Another objective of this study was to investigate the stability of a mosaicplasty during motion (with or without weight). For the whole group (unbottomed and bottomed together), neither motion protocols significantly changed surface congruency; it remained equal to the mosaicplasty directly after surgery. This can be concluded from the fact that the pressure patterns did not change after weight 
bearing or non weight bearing motion, as the pressure pattern and rim stress remained equal. When comparing the bottomed and unbottomed groups, two unbottomed mosaicplasties showed a pressure pattern according to subsidence below flush level after weight bearing motion. Since the main focus of this study was to measure peak stresses on the articular surface, we did not assess the actual amount of subsidence. Although no significant differences in contact stresses could be detected between bottomed and unbottomed plugs by comparing the total groups; one can at least have some concern about potential subsidence of unbottomed plugs. Nevertheless, most unbottomed plugs remained stable, which is in agreement with a study of Pearce et al. [15], who reported that unbottomed multiple plug mosaicplasties that were placed flush, continued to stay flush after 3 months of weight bearing motion in sheep. However, human bone is much softer and therefore it may be more difficult to obtain a stable reconstruction with unbottomed plugs. In our previous study with human femora we measured the force required to push a single plug below flush level. For an unbottomed plug of $16 \mathrm{~mm}, 151 \mathrm{~N}$ had to be applied in comparison with $294 \mathrm{~N}$ for the bottomed plug [8]. For the comparison with the multiple plug mosaicplasty, Kordas et al. [10] reported that application of a mean force of $54 \mathrm{~N}$ was enough to push an unbottomed multiple plug mosaicplasty $3 \mathrm{~mm}$ below flush level; unfortunately, there was not a bottomed comparison in that study. The applied force of $350 \mathrm{~N}$ during the weight bearing motion in our study may have been too low, to destabilize the reconstruction. This load, which is relatively low, was chosen, as it would simulate partial weight-bearing motion during the direct postoperative rehabilitation period and not level walking. The latter is, according to our protocol, permitted after 24 weeks, which should allow for in-growth of the plugs $[11,15]$.

\section{Clinical relevance}

A cartilage defect results in increased stress levels at the articular cartilage boundaries of the defect. A multiple plug mosaicplasty has a positive effect in reducing these stress elevations, which will reduce the potential of cartilage degeneration, and thus may postpone secondary osteoarthritis. Post-operative non-weight bearing and weight bearing motions did not seem to influence the surface congruency of the mosaicplasty, although the unbottomed mosaicplasties showed a trend of subsidence below flush level after weight bearing motion. It appears feasible to allow limited weight bearing of the knee after osteochondral transplantation, especially when plugs have been bottomed.
Open Access This article is distributed under the terms of the Creative Commons Attribution Noncommercial License which permits any noncommercial use, distribution, and reproduction in any medium, provided the original author(s) and source are credited.

\section{References}

1. Barink M, Meijerink H, Verdonschot N, Van Kampen A, de Waal Malefijt M (2007) Asymmetrical total knee arthroplasty does not improve patella tracking: a study without patella resurfacing. Knee Surg Sports Traumatol Arthrosc 15:184-197

2. Brimacombe JM, Anglin C, Hodgson AJ, Wilson DR (2005) Validation of calibration techniques for tekscan pressure sensors. In: Proceedings of international society of biomechanics XXth congress. ASB 29th Annual meeting, Cleveland, p 263

3. Brown TD, Pope DF, Hale JE, Buckwalter JA, Brand RA (1991) Effects of osteochondral defect size on cartilage contact stress. J Orthop Res 9:559-567

4. Guettler JH, Demetropoulos CK, Yang KH, Jurist KA (2004) Osteochondral defects in the human knee: influence of defect size on cartilage rim stress and load redistribution to surrounding cartilage. Am J Sports Med 32:1451-1458

5. Hangody L, Kish G, Karpati Z, Udvarhelyi I, Szigeti I, Bely M (1997) Autogenous osteochondral graft technique for replacing knee cartilage defects in dogs. Orthopedics 5:175-171

6. Hangody L, Feczko P, Bartha L, Bodo G, Kish G (2001) Mosaicplasty for the treatment of articular defects of the knee and ankle. Clin Orthop Relat Res 391(Suppl):S328-S336

7. Jackson DW, Lalor PA, Aberman HM, Simon TM (2001) Spontaneous repair of full-thickness defects of articular cartilage in a goat model: a preliminary study. J Bone Joint Surg 83:53

8. Kock NB, van Susante J, Buma P, Van Kampen A, Verdonschot N (2006) Press-Fit Stability of an osteochondral autograft: influence of different plug length and perfect depth alignment. Acta Orthop 77:422-428

9. Koh JL, Wirsing K, Lautenschlager E, Zhang LO (2004) The effect of graft height mismatch on contact pressure following osteochondral grafting: a biomechanical study. Am J Sports Med $32: 317-320$

10. Kordas G, Szabo J, Hangody L (2006) Primary stability of osteochondral grafts used in mosaicplasty. Arthroscopy 22: $414-421$

11. Lane JM, Brighton CT, Ottens HR, Lipton M (1977) Joint Resurfacing in the rabbit using an autologous osteochondral graft. J Bone Joint Surg 59:218-222

12. Lefkoe TP, Trafton PG, Ehrlich MG, Walsh WR, Dennehy DT, Barrach H-J, Akelman E (1993) An experimental model of femoral condylar defect leading to osteoarthrosis. J Orthop Trauma 7:458-467

13. Linden B (1977) Osteochondritis dissecans of the femoral condyles: a long-term follow-up study. J Bone Joint Surg 59: 769-776

14. Messner K, Maletius W (1996) The long-term prognosis for severe damage to weight-bearing cartilage in the knee: a 14-year clinical and radiographic follow-up in 28 young athletes. Acta Orthop 67:165-168

15. Pearce SG, Hurtig MB, Clarnette R, Kalra M, Cowan B, Miniaci A (2001) An investigation of 2 techniques for optimizing joint surface congruency using multiple cylindrical osteochondral autografts. Arthroscopy 17:50-55

16. Pena E, Calvo B, Martinez MA, Doblare M (2007) Effect of the size and location of osteochondral defects in degenerative arthritis. a finite element simulation. Comput Biol Med 37: 376-387 
17. Raimondi MT, Peitrabissa R (2005) Contact pressures at grafted cartilage lesions in the knee. Knee Surg Sports Traumat Arthrosc V13:444-450

18. van Susante LC, Wymenga B, Buma P (2003) Potential healing benefit of an osteoperiosteal bone plug from the proximal tibia on a mosaicplasty donor-site defect in the knee. Arch Orthop Trauma Surg V123:466-470

19. Tienen TG, Verdonschot N, Heijkants RGJC, Buma P, Scholten JGF, van Kampen A, Veth RPH (2004) Prosthetic replacement of the medial meniscus in cadaveric knees: does the prosthesis mimic the functional behavior of the native meniscus? Am J Sports Med 32:1182-1188

20. Whiteside RA, Bryant JT, Jakob RP, Mainil-Varlet P, Wyss UP (2003) Short-term load bearing capacity of osteochondral autografts implanted by the mosaicplasty technique: an in vitro porcine model. J Biomech 36:1203-1208

21. Wu JZ, Herzog W, Hasler EM (2002) Inadequate placement of osteochondral plugs may induce abnormal stress-strain distributions in articular cartilage - finite element simulations. Med Eng Phys 24:85-97 